\title{
Heartbeat: bathing daily is associated with a lower
} cardiovascular risk

Lifestyle behaviours such as smoking, exercise and diet have received consideration attention as risk factors for cardiovascular disease (CVD) with lifestyle modification now serving as the foundation for primary prevention. Tub bathing is a potentially beneficial behaviour that has received less attention, possibly due to geographic and cultural variation in this behaviour. Japanese tub bathing, which typically involves sitting in hot water to shoulder depth, exposes the bather both to heat and to water pressure, which results in an increase in stroke volume and cardiac output with a reduction in systemic vascular resistance. Ukai and colleagues ${ }^{1}$ investigated the association between tub bathing and long-term CVD risk in a study of over 30000 participants without CVD at baseline who were then followed for 20 years ${ }^{1}$. On multivariable analysis, the risk of total CVD was lower in those who bathed almost daily or daily compared with those who bathed two or fewer times per week (HR 0.72, 95\% CI 0.62 to 0.84 , trend $\mathrm{p}<0.001)$ with similar trends for coronary artery disease and stroke risk (figure 1).

In the accompanying editorial, Burden ${ }^{2}$ cautions that confounding variables may account for the observed associations, despite the author's attempt to account for known differences between groups. In addition, the style of bathing in Japan, sitting with hot water to shoulder height, sadly is not available to most of us worldwide. Finally, the study group was relatively young (age 40-59 years) and the higher risk of sudden death with hot bathing in older adults may offset any potential benefit. Burden concludes: 'Investigations into the potential cardiovascular benefit of head-free immersion in warm to hot water are needed. In the meanwhile, caution is needed because of the high mortality associated with such bathing in an unselected population.'

In patients with coronary disease, the benefit of revascularisation when the fractional flow reserve (FFR) is intermediate $(0.75-0.80)$, or in the 'grey zone', remains

Correspondence to Professor Catherine M Otto, Division of Cardiology, University of Washington, Seattle,WA 98195, USA; cmotto@uw.edu

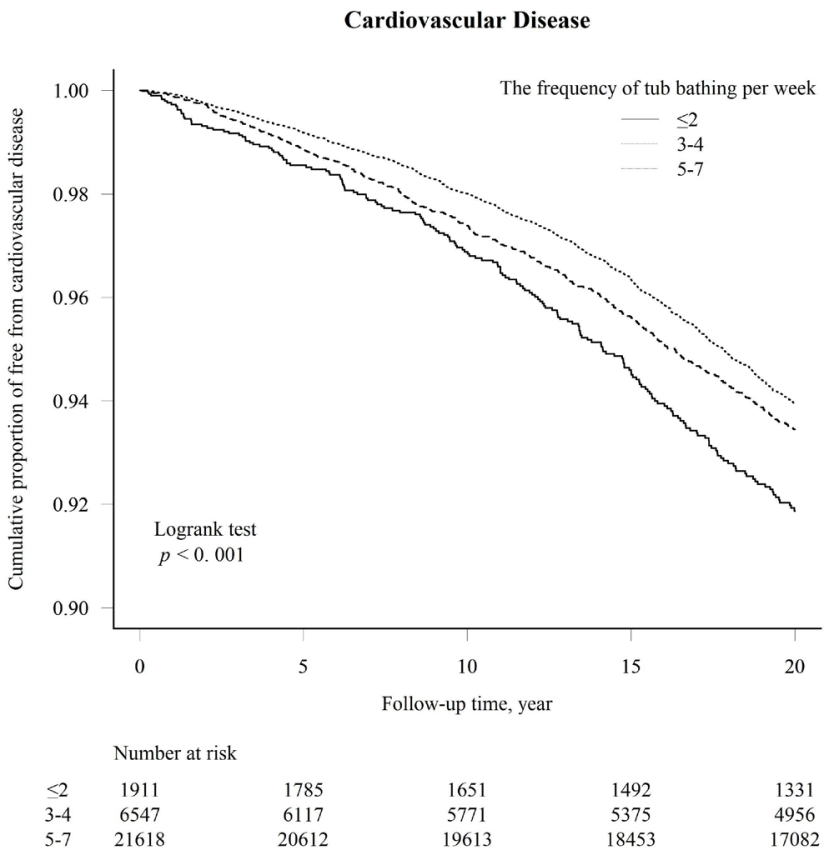

Figure 1 Cumulative Kaplan-Meier curve for proportion of free from cardiovascular disease according to the frequency of tub bathing per week.

controversial. In order to determine the effects of percutaneous coronary intervention (PCI) on symptoms in patients with a grey-zone FFR, Hennigan and colleagues ${ }^{3}$ randomised 104 symptomatic patients with a $30 \%-80 \%$ coronary stenosis and an FFR of $0.75-0.80$ to optimal medical therapy (OMT) alone or PCI plus OMT

(figure 2). At 3 months follow-up, those randomised to PCI had fewer angina symptoms (21 (28) vs $10(23) ; \mathrm{p}=0.026)$ and a better quality of life (24 (26) vs 11 (24); $\mathrm{p}=0.008)$ than those treated with OMT alone. However, by 1 year, there was no significant difference between groups in either outcome.

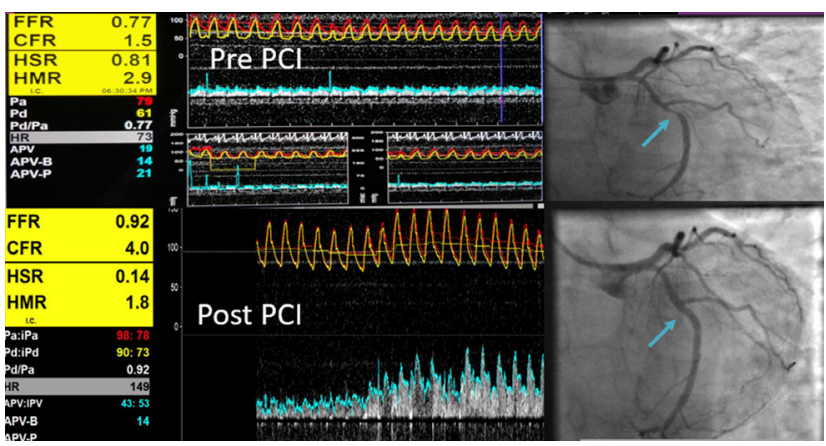

Figure 2 This patient had a moderately severe mid left circumflex lesion with ${ }^{\mathrm{GZ}} \mathrm{FFR}$ physiology with reduced CFVR of 1.5 pre-PCI which improved to a CFVR of 4 post- $\mathrm{PCl}$. The stenosis resistance $\mathrm{HSR}$ reduced following $\mathrm{PCl}$ with improved FFR. ${ }^{\mathrm{GZ}} \mathrm{FFR}$ coronary lesion in mid circumflex indicated by blue arrow before $\mathrm{PCI}$ (upper panel) and after $\mathrm{PCI}$ with coronary physiology data in the left panel. CFR, coronary flow reserve; FFR, fractional flow reserve; ${ }^{G Z} F F R$, grey zone; HSR, hyperaemic stenosis resistance index; $\mathrm{PCl}$, percutaneous coronary intervention, $\mathrm{HMR}$, hyperaemic microvascular resistance. 


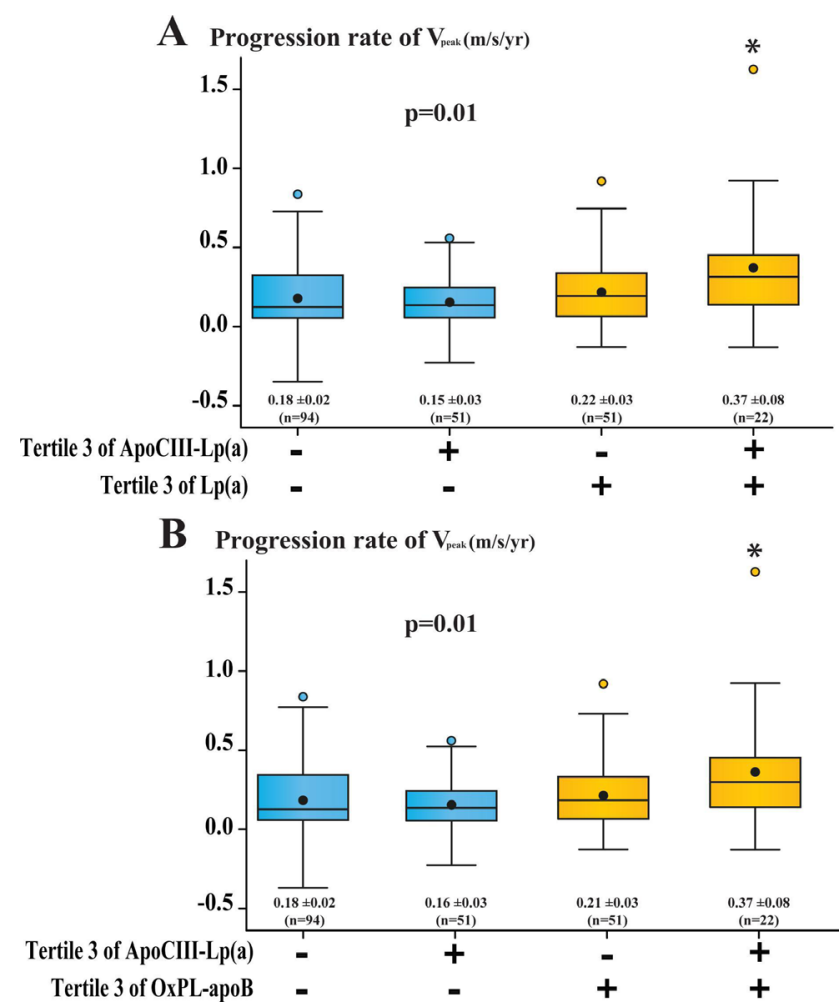

Figure 3 AS progression rate according to top tertiles of ApoCIII-Lp(a) and Lp(a) or OxPL-apoB. Comparison of annualised Vpeak after dichotomisation by middle and bottom tertiles versus top tertile of ApoCIII-Lp(a) (ie, ApoCIII-Lp(a) $\leq$ vs $>297000$ RLU) and tertile of $L p(a)$ (ie, interaction between tertiles of $A p o C I I I-L p(a)$ and $L p(a))(A)$ or tertile of OxPL-apoB (ie, interaction between tertiles of ApoCIII-Lp(a) and OxPL-apoB) (B). ApoCIII, apolipoprotein C-III; Lp(a), lipoprotein(a); Vpeak, peak aortic jet velocity. $P$ values are from two-way ANOVA. ${ }^{*} \mathrm{P}<0.05$ compared with the other three groups (from Tukey's post hoc test). The box shows 25th and 75th percentiles, the median line shows the median value and the black dot the mean value, error bars the 10th and 90th percentiles; circles are outliers. The numbers at the bottom of the graph are mean \pm SEM of the progression rate of Vpeak and between brackets the number of patients in each group. ApoCIII, apolipoprotein CIII; apoB, apolipoprotein B; ANOVA, analysis of variance; AS, aortic valve stenosis; Lp(a), lipoprotein(a); OxPL, oxidised phospholipids; RLU, relative light units.

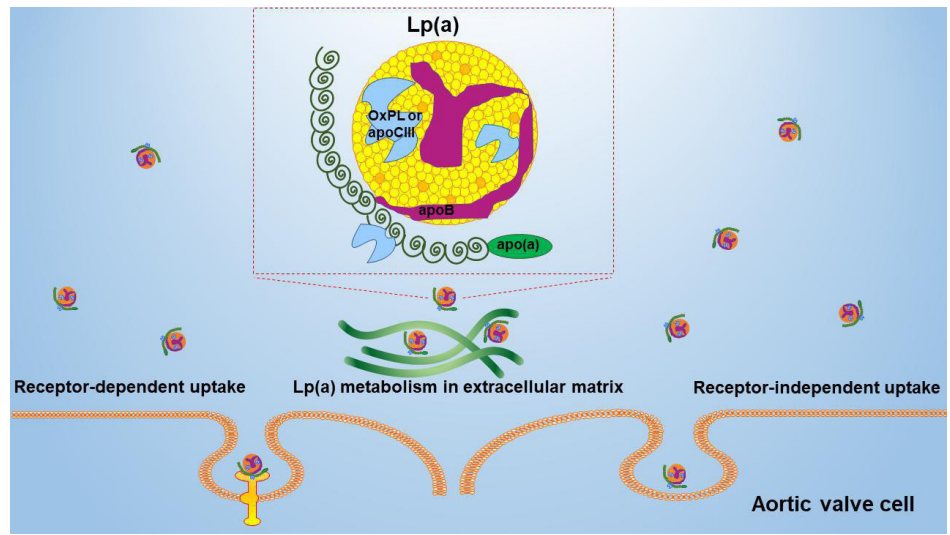

Figure $4 \mathrm{Lp}(\mathrm{a})$ complexes, metabolism and uptake in the aortic valve. Complexing of apo(a) and apolipoprotein B with OxPLs or apoCIII in a subset of $\mathrm{Lp}(\mathrm{a})$ particles may alter the metabolism and uptake of $\mathrm{Lp}(\mathrm{a})$ or molecules contained in or produced from $\mathrm{Lp}(\mathrm{a})$ particles, stimulating procalcific mechanisms and aortic stenosis. Lp(a) may be taken up via receptor-dependent or receptor-independent means, along with being metabolised in the extracellular matrix, leading to the release of molecules that are subsequently taken up by valve cells. apo(a), apolipoprotein(a); apoCIII, apolipoprotein CIII; Lp(a), lipoprotein(a); OxPL, oxidised phospholipid.
Ihdayhid and Sapontis ${ }^{4}$ comment that: 'The FFR grey zone has served cardiology well. In advocating for clinical judgement, the consideration of additional information and the avoidance of a dichotomous way of thinking, the grey zone serves as a blueprint for the future paradigm of coronary revascularisation. As we move into an era that will increasingly question the rationale for revascularisation in stable $\mathrm{CAD}$, it is important that we expand the concept of the grey zone to many of our treatment decisions. As highlighted by the results of this elegant $s^{2} \mathrm{dy}^{3}$,the benefits of our interventions can be shrouded in shades of grey.'

Calcific aortic stenosis (AS) is the endstage of an active disease process, yet approaches to slowing progression of disease in the valve leaflets have been elusive. Based on previous genetic and basic science studies, Capoulade and colleagues ${ }^{5}$ hypothesised that apolipoprotein CIIIlipoprotein(a) complexes (ApoCIII-Lp(a)) would be associated with the rate of progression of calcific AS. In 218 patients with initially mild-moderate AS, patients with higher circulating levels of ApoCIII$\mathrm{Lp}(\mathrm{a})$ and $\mathrm{Lp}(\mathrm{a})$ had a significantly faster rate of haemodynamic progression and were at higher risk of valve replacement or death, comparing those in the top tercile to the remainder of the study group, even after multivariable adjustment. Similar associations between serum levels of other lipoprotein complexes and rapid disease progression as shown in figure 3 .

The nuances of the association between lipoprotein complexes and calcific AS are discussed in detail by Rogers and Aikawa ${ }^{6}$ who suggest that 'Clinical studies assessing the effectiveness of $\operatorname{Lp}(\mathrm{a})$ or ApoCIII antisense oligonucleotides, EO6 antibodies or other means of targeting these apolipoproteins and oxidised lipids could be the next major therapeutic step to identify non-surgical interventions for calcific aortic valve disease, particularly in the high-risk population of patients with elevated Lp(a).'(figure 4)

A comprehensive statement from the British Association for Cardiovascular Prevention and Rehabilitation (BACPR) provides a summary and recommendations for optimal nutritional strategies for primary and secondary prevention of CVD. ${ }^{7}$ They recommend switching the focus from macronutrients (protein, carbohydrate, etc) to whole foods (fruits, vegetables, eggs, meat, etc) when providing dietary advice to patients. Both healthcare providers and patients will appreciate the clear, concise and practical advice in this detailed document. 
The Education in Heart article in this issue $^{8}$ summarises the approach to the interpretation and management of narrow complex tachyarrhythmias.

Contributors Heartbeat for Heart 106 Issue 10.

Funding The authors have not declared a specific grant for this research from any funding agency in the public, commercial or not-for-profit sectors.

Competing interests None declared.

Patient and public involvement Patients and/ or the public were not involved in the design, or conduct, or reporting, or dissemination plans of this research.

Patient consent for publication Not required.

Provenance and peer review Not commissioned; internally peer reviewed.
(C) Author(s) (or their employer(s)) 2020. No commercial re-use. See rights and permissions. Published by BMJ.

\section{(D) Check for updates}

To cite Otto CM. Heart 2020;106:707-709.

Heart 2020;106:707-709.

doi:10.1136/heartjnl-2020-317029

ORCID iD

Catherine M Otto http://orcid.org/0000-0002-05279392

\section{REFERENCES}

1 Ukai T, Iso H, Yamagishi K. Habitual tub bathing and risks of incident coronary heart disease and stroke. Heart 2020;106:732-7.

2 Burden AF. Tub bathing and heart disease. Heart 2020;106:710.
3 Hennigan B, Berry C, Collison D, et al. Percutaneous coronary intervention versus medical therapy in patients with angina and grey-zone fractional flow reserve values: a randomised clinical trial. Heart 2020;106:758-64.

4 Indayhid AR, Sapontis J. The fractional flow reserve grey zone: a blueprint for the future of coronary revascularisation. Heart 2020;106:714-5.

5 Capoulade R, Torzewski M, Mayr M, et al. ApoCIII-Lp(a) complexes in conjunction with Lp(a)-OxPL predict rapid progression of aortic stenosis. Heart 2020;106:738-45.

6 Rogers MA, Aikawa E. Complex association of lipoprotein(a) with aortic stenosis. Heart 2020;106:711-2.

7 Butler T, Kerley CP, Altieri N, et al. Optimum nutritional strategies for cardiovascular disease prevention and rehabilitation (BACPR). Heart 2020;106:724-31.

8 Badhwar N. Approach to narrow complex tachycardia: non-invasive guide to interpretation and management. Heart 2020;106:722-83. 